MODELING, IDENTIFICATION AND CONTROL, 1980, vOL. 1, NO. 2, 69-91

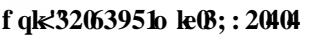

\title{
A model of the dynamics of plankton patchiness
}

\author{
WOLFGANG EBENHÖH††
}

Keywords: modelling, phytoplankton, zooplankton, patchiness, simulation, ecosystem, density distribution, spatial distribution.

A mathematical model of the dynamics of plankton patchiness in the intermediate scale $(1 \mathrm{~km}-10 \mathrm{~km})$ was developed. Mechanisms that may be important in the creation and destruction of patches were selected and modelled. Such mechanisms are: horizontal turbulent diffusion, noise in the vertical turbulence, vertical migration of the zooplankton combined with a velocity profile and consumption of zooplankton by fish in schools. Patchiness is described by the use of the moments of density distributions, coherence lengths and correlations of phytoplankton and zooplankton. These parameters are investigated as functions of time and, also, for their dependence on the parameters of the patch creation mechanisms.

\section{Introduction}

\subsection{The role of plankton patchiness in the marine ecosystem}

Plankton is very inhomogeneously distributed in space and time. Until recently, the theoretical investigations of the plankton system were concentrated on the inhomogeneity in time and on the inhomogeneity in space in the vertical direction. From recent studies, investigators have an improved understanding of the dynamics of horizontally homogeneous plankton systems and of its vertical structure (e.g. Parsons et al. 1977). The situation is quite different for the horizontal spatial inhomogeneity of the plankton distribution, commonly called patchiness. (In the following, we use the term 'homogeneous' as referring to horizontal homogeneity.) Only recently has patchiness come to be of central interest to marine scientists (e.g. Steele 1974, Cushing and Walsh 1976, Platt 1975, Wroblewski and O'Brien 1976, Dubois and Closset 1975).

Patchiness can be found on any scale. In this paper, we deal with patchiness in the intermediate scale of $1 \mathrm{~km}-10 \mathrm{~km}$. Below $100 \mathrm{~m}$, the horizontal turbulence leads to short life times of spatial structures (Platt et al. 1975). Above $100 \mathrm{~km}$, the physical oceanographic factors are dominant. Such factors are upwelling currents determined by the geographics of the bottom of the ocean (Wroblewski 1976), the proximity of the coastline or the icecover, salinity changes by rivers, wells, or ice. But these 'deterministic' inhomogeneities of the plankton distribution will not be considered here. In contrast to this, the spatial structure in the intermediate scale has a strong stochastic element. Turbulence and currents produce an interaction of plankton in different locations. Due to interference and cooperation of physical factors with biological factors, in this intermediate scale, spatial structures are created and destroyed. The scale of these inhomogeneities does not agree with the scale of change of the physical

$\dagger$ Universität Oldenburg, Fachbereich IV, Mathematik Oldenburg, Germany.

$\ddagger$ This work was done during the author's stay at The Norwegian Institute of Technology, Division of Engineering Cybernetics, on leave of absence from the University of Oldenburg, Department of Mathematics, Oldenburg, Germany. 
factors, as it does in the case of large scale patchiness (Kierstead and Slobodkin 1953, Platt 1975). Furthermore, Platt (1972) has determined a power spectrum of the phytoplankton distribution. He found that the most important contributions to the variance come from the $1 \mathrm{~km}$ range.

On the biological side, patchiness, in the $1 \mathrm{~km}$ range, is, very probably, fundamentally important in the coupling of the plankton system to the fish system (Steele 1974). In Fig. 1, the connections between the plankton system and other subsystems of the marine ecosystem are presented. As can be seen, patchiness plays an essential role in all the connections. For an ecosystem model, the plankton submodel has to be a patchiness model.

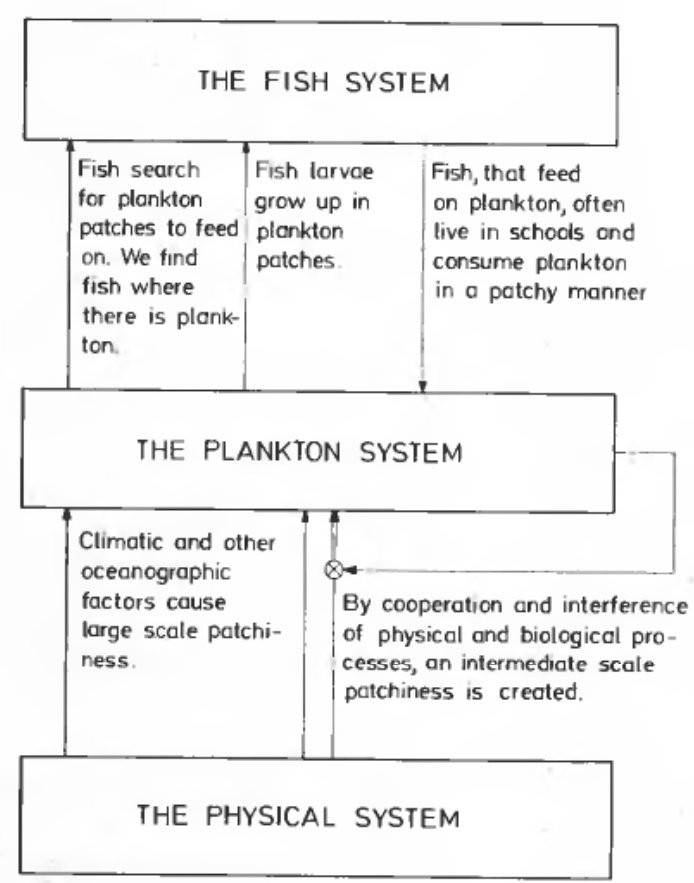

Figure 1. Coupling of the plankton system to other subsystems of the marine ecosystem. For these couplings, the patchiness is an essential feature of the plankton system.

\subsection{Description of patchiness}

It is clearly impossible and uninteresting to describe position, size, form and character of the single patches. In addition to the variables from a homogeneous plankton system, a set of few variables, describing patchiness in a global way, has to be constructed. They should be such that:

-they are directly useful to the fish system;

-they are convenient for the coupling to the physical system;

- a dynamic model can be constructed for them;

- an adequate description of patchiness, with a minimal numter of variables, is possible. 
A straightforward idea would be the description of the patchy structure by the variables:

mean area of patches,

mean distance between patches,

mean density inside and outside the patches.

Such a description could be convenient for most purposes but is not an adequate description of real patchiness. Contour maps of plankton distributions show very irregular forms.

A much better idea is the construction of an auto-correlation function and the derivation of a power spectrum. Platt and Denman (1975) describe their power spectra by the use of only 3 to 4 parameters. Patchiness description, in this way, is attractive because it is adequate and the coupling to the physical system is possible (Denman et al. 1977).

On the other hand, however, with the power spectrum parameters as additional plankton state variables, it is not easy to couple the plankton system to the fish system; and to model their dynamics (time-dependence) seems to be extremely difficult. Thus, it is a complicated task to select patchiness parameters, fulfilling the criteria above. Some experience with the plankton model, presented in this paper, together with progress on a fish model, will help to find the best solution.

An excellent review on the mathematical analysis of plankton patchiness can be found in Fasham (1978).

\subsection{The structure and the task of the plankton model}

The patchiness problem is approached in a very direct way. A simple homogeneous plankton model system is constructed, $(\S 2)$, and from 400 such building blocks a plankton model is assembled. The homogeneous plankton subsystems are arranged in a $20 * 20$ array. For simplicity, the rectangular array of subsystems is periodically continued to infinity; that produces a two-dimensional torus. Then, one never has to contend with edge problems. The subsystems interact due to turbulent diffusion, due to currents (which may be different in upper and lower waterlayers), and due to the movement of schools of fish within this model ocean. In addition, distortions with stochastic properties (noise) act on the homogeneous subsystems (§3).

A first task of the present model will be the development of patchiness variables. The general objective is, however, the investigation of the mechanism which create and destroy the patchy structure of the plankton distribution within the intermediate scale.

\section{The homogeneous plankton subsystem}

\subsection{The state variables}

Not all the detailed features of the homogeneous plankton subsystems are critically essential for organization and behaviour of the composed system. This is a general feature of models with well-separated organization levels (hierarchical structure). Hence, even with very simplified models as building elements, the essentials of the composed model can be investigater. With the three variables:

$N$ : nutrient concentration

$P$ : phytoplankton density

$Z$ : zooplankton density,

it is possible to construct a plankton submodel, sufficient for the purpose. An ensemble 
of such subsystems should be coupled to the physical processes and to the fish system and it should allow studies of the patch creation mechanisms.

There are several shortcomings of such a simplified submodel in comparison to reality and to the well-developed models of Slagstad (1980) and Greve (1977), (see also Sonntag and Greve 1977): age structure and size distribution of the zooplankton, the vertical structure of the plankton distribution, and the species' diversity is neglected; a carnivore zooplankton component is insufficiently treated; and 'nutrient' stands for the soluble nitrogen compounds, neglecting phosphate and silicate. Without a proper size distribution of the zooplankton, the survival fight of the fishlarvae may not be considered. This is the severest shortcoming of the simplified submodel. The species' diversity generally causes greatest difficulties to the model builders. Certainly, it plays an important role for the stability of the ecosystem against all kinds of external impacts.

\subsection{The dynamic equations}

The homogeneous submodel is constructed to fulfil dynamic equations of the form:

$$
\begin{aligned}
& \dot{N}=-\lambda G_{P}(N) P+\text { Recycling terms }+\kappa_{V}\left(N_{0}-N\right) \\
& \dot{P}=\lambda G_{P}(N) P-\alpha G_{Z}(P) Z-\kappa_{V} P \\
& \dot{Z}=v_{Z} \alpha G_{Z}(P) Z-\beta G_{F}(Z)-\sigma_{Z} Z
\end{aligned}
$$

The first term of the r.h.s. in eqn. (2) is the phytoplankton growing term. It appears negative in eqn. (1) without an additional factor. This is because we measure nutrients $N$, the phytoplankton density $P$, and the zooplankton density $Z$, all in nitrogen equivalents $\mu M N / l$. The second term in eqn. (2) describes the consumption of phytoplankton. A part $v_{Z}$ of this term appears as an increase of the zooplankton density in eqn. (3). The other part $\left(1-v_{Z}\right)$ is excreted. A part $\mu_{Z}$ of the excreted nitrogen is released in soluble form in the euphotic zone and makes up a recycling term in eqn. (1). The rest leaves the system. The second term in eqn. (3) describes the mortality of the zooplankton due to fish and due to carnivorous zooplankton. As before, a part $\mu_{F}$ of this term forms a second recycling term.

For all the terms considered up to now, the same structure is assumed: term $=$ rate constant $*$ saturation function*predator density

The saturation functions $G$ depend on the densities of the prey species and are normalized to 1 . For $G_{P}(N)$ we assume a Michaelis-Menten form with Michaelis constant $N_{M}$, while modified Ivlev forms are chosen for $G_{Z}$ and $G_{F}$ (Parsons, Lebrasseur and Fulton 1967, O’Brien and Wroblewski 1973, Wroblewski 1976):

$$
\begin{aligned}
& G_{Z}(P)= \begin{cases}0 & \text { for } P \leq P_{T} \\
1-\exp \left(-\left(P-P_{T}\right) / P_{M}\right) & \text { for } P>P_{T}\end{cases} \\
& G_{F}(Z) \text { corresponding. }
\end{aligned}
$$

The constants $P_{T}, Z_{T}$ are feeding threshold levels, while the constants $P_{M}, Z_{M}$ are connected to the half-saturation densities $\left(P_{T}+P_{M} \ln 2\right)$.

The last terms in the eqns. (1) to (3) are purely exponential terms. Most important is the nutrient source term $\kappa_{V}\left(N_{0}-N\right)$ where $N_{0}$ is the nutrient concentration below the euphotic zone and $\kappa_{V}$ is a constant describing the vertical turbulent diffusion. The 
same process that brings up nutrient-rich water in the euphotic zone dilutes the phytoplankton concentration there $\left(-\kappa_{V} P\right)$. An analogue term is added for $\dot{Z}$ in eqn. (3). It describes the zooplankton mortality due to starvation in winter and is unimportant during the food-rich seasons.

The phytoplankton growing parameter $\lambda$ is taken to be time dependent in such a way that it is proportional to the length of the daylight which changes with the season:

$$
\lambda=\lambda_{0} \frac{\text { length of the daylight }}{24 \mathrm{~h}}
$$

\subsection{The parameter values}

In Table 1, the parameters of the homogeneous submodel are collated. The choice of the values was guided by comparison with Steele (1974), Wroblewski (1976), and Slagstad (1979), where related, but more complicated, models, with other aims, can be found. Because a comprehensive ecological model for the Barents Sea is under construction (Balchen 1979 a), some model parameters are fitted to the Barents Sea (e.g. $N_{0}$ as low as $10 \mu M N / l$, and $24 \mathrm{~h}$ as maximal length of daylight). The dynamics are influenced most strongly by the parameters $\lambda_{0}, \alpha, \nu_{Z}, \kappa_{V}$, and $\beta$. While $\lambda_{0}$ determines essentially the total time scale, $\alpha$ and $\nu_{Z}$ influence the relationship between phytoplankton and zooplankton. The vertical turbulence parameter $\kappa_{V}$ determines the character of the plankton oscillations which become undamped if $\kappa_{V}$ is high.

In the context of this model, the zooplankton mortality is of special importance (§3.2). It is not easy to measure and, hence, not well known. The constant $\beta$ is assumed to be a combination of two contributions:

$$
\beta=\beta_{\mathrm{c}}+\beta_{\mathrm{F}} ; \quad q_{\mathrm{F}}=\beta_{\mathrm{F}} / \beta
$$

The carnivorous zooplankton (e.g. ctenphores) is not a state variable in the model, it acts only as a constant mortality $\beta_{\mathrm{c}}$ on $Z$. Another fraction $q_{F}=\beta_{F} / \beta$ of the total mortality is due to fish that feed on plankton. The total number of fish is also considered as constant (but compare § 3.2). For simplicity, the same saturation function $G_{F}(Z)$ for both parts of the zooplankton mortality is assumed.

The dynamics of the homogeneous plankton subsystem depend, in a strongly nonlinear way, on the parameter $\beta$. This feature is, however, not a property of just these model eqns. (1) to (3); it will be found in many related models. To clarify the role of this nonlinearity, the surface $\dot{N}=0$ in the three-dimensional state space $(N, P, Z)$ is projected on the $P, Z$-plane (Fig. 2). The crosspoints of the lines $\dot{P}=0$ and $\dot{Z}=0$ depict the stationary states, depending on the parameter combinations. In Fig. 2 , the points $\mathrm{A}$ and $\mathrm{C}$ correspond to stable stationary states with very different ratios between phytoplankton and zooplankton, while the system will show undamped oscillations around $\mathrm{B}$.

Thus, even moderate changes of the zooplankton mortality $\beta$ can lead to drastic changes of the character of the steady state. This can be further demonstrated by the time course of the three variables $N, P, Z$ (Fig. 3 ). The oscillations of the submodel in Fig. 3 can easily be damped by the use of a smaller vertical turbulence $\kappa_{V}$.

\section{Interaction between the homogeneous subsystems and the creation of patches}

\subsection{Diffusion}

The horizontal turbulent diffusion is the best investigated physical process involved in the patch dynamics. The information about this process is available due to the 


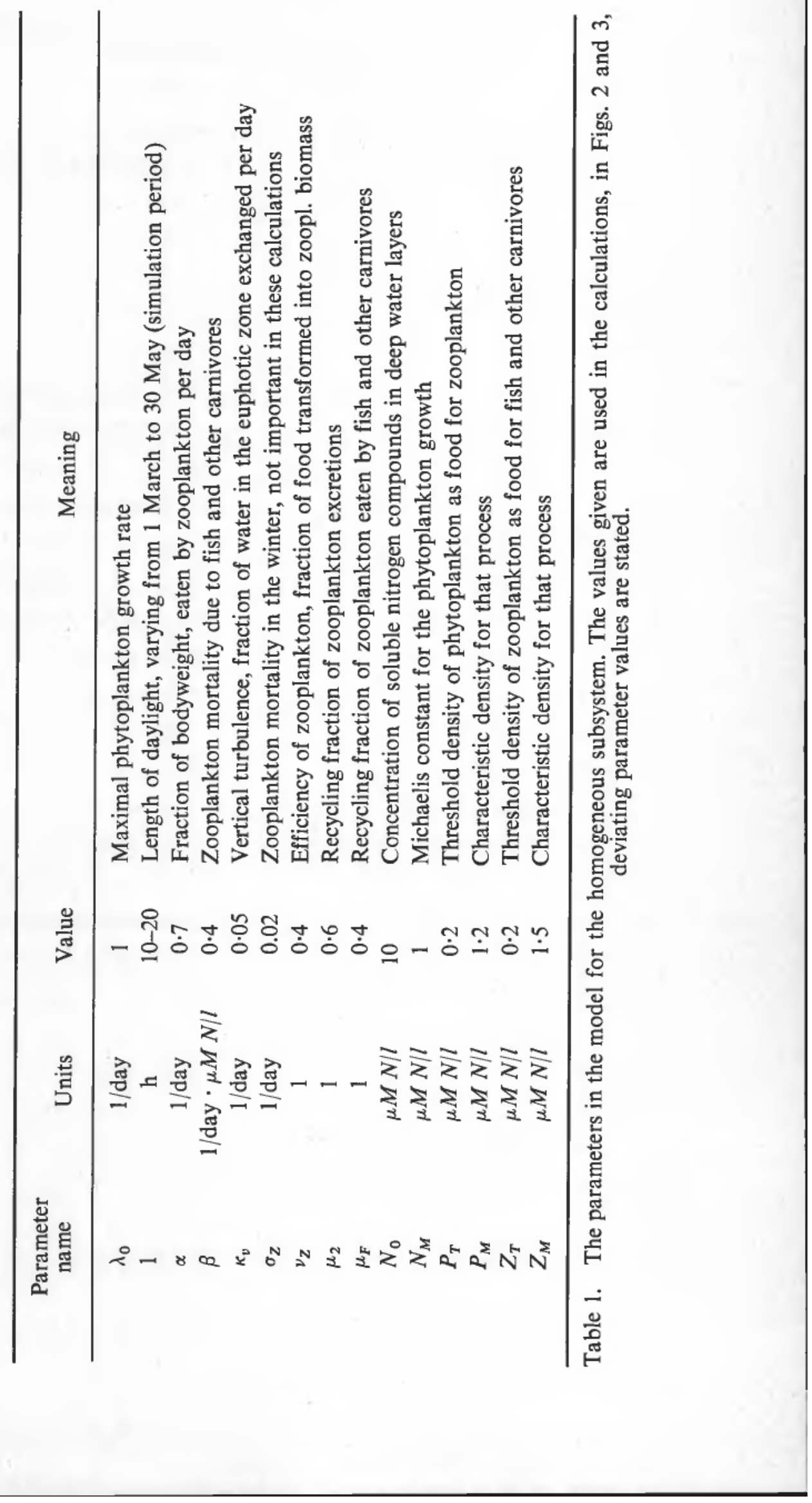




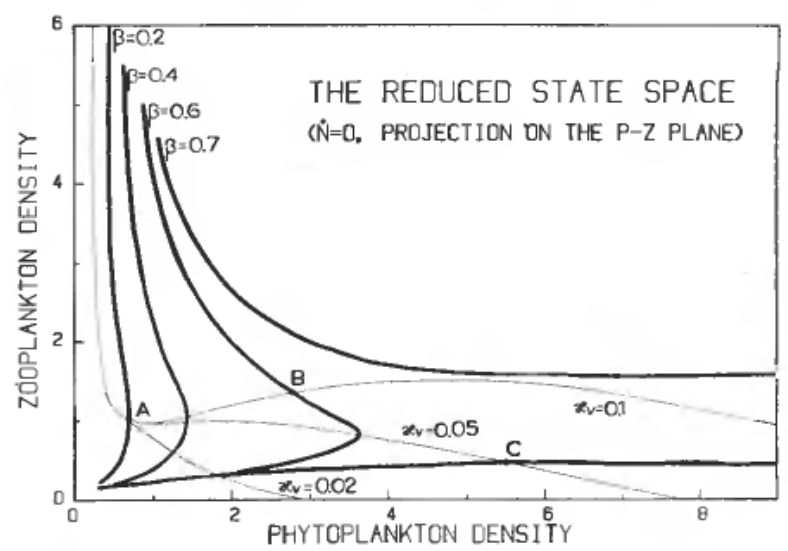

Figure 2. The reduced state space (surface $\dot{N}=0$ ). The crosspoints of the lines $\dot{P}=0$ (thick) and $\dot{Z}=0$ (thin), e.g. A, B, C, depict the steady states depending on the parameters $\beta$ (zooplankton mortality) and $\kappa_{V}$ (vertical turbulence). For units and other parameters see Table 1.

observation of the decay of oil and colour patches. Each single patch undergoes an extremely complicated deformation. An adequate description of the form is probably possible only with the fractals of Mandelbrot (1977). But on the average, the dispersion process due to the horizontal turbulences may be described in analogy to the molecular diffusion by

$$
\dot{P}(x, y, t)=\kappa \Delta P(x, y, t)
$$

where $P(x, y, t)$ is the density of the dispersed material. It turns out that such an assumption requires a scale-dependent, horizontal diffusion constant $\kappa$. For the ocean, Okubo (1971) has determined this scale dependence of $\kappa$ to

$$
\kappa(L)=\kappa\left(L_{0}\right) \cdot\left(\frac{L}{L_{0}}\right)^{1 \cdot 15}
$$

where $L$ is the 'scale' of the diffusion process and where $L_{0}$ is a reference scale:

$$
L_{0}=1 \mathrm{~km}, \kappa\left(L_{0}\right) \cong 0.05 \mathrm{~km}^{2} / \text { day. }
$$

Of great theoretical interest is the power 1.15 but so far there is no satisfactory explanation for it.

In the plankton model, which is an array of 400 submodels, the r.h.s. of eqn. (5) has to be substituted by an expression containing the finite distance between the centres of the neighbouring submodels:

$$
\begin{aligned}
\dot{P}(i, j) & =\kappa_{H}(P(i+1, j)+P(i-1, j)+P(i, j+1)+P(i, j-1)-4 P(i, j)) \\
\kappa_{H} & =\kappa(L) / L^{2}
\end{aligned}
$$

Here, the integers $i$ and $j$ give the location of the submodel in the array and $L$ has the twofold meaning: diameter of the subsystems and scale of the process. The constant $\kappa_{H}$ on the r.h.s. of eqn. (6) depends on $L$ like $L^{-0.85}$. For small scales, this term will override any counteracting process and will lead to short lifetimes of small patches. For an array of $L=1 \mathrm{~km}$ submodels, one obtains from Okubo's data (1971) the number $\kappa_{H}=0 \cdot 05 /$ day. 

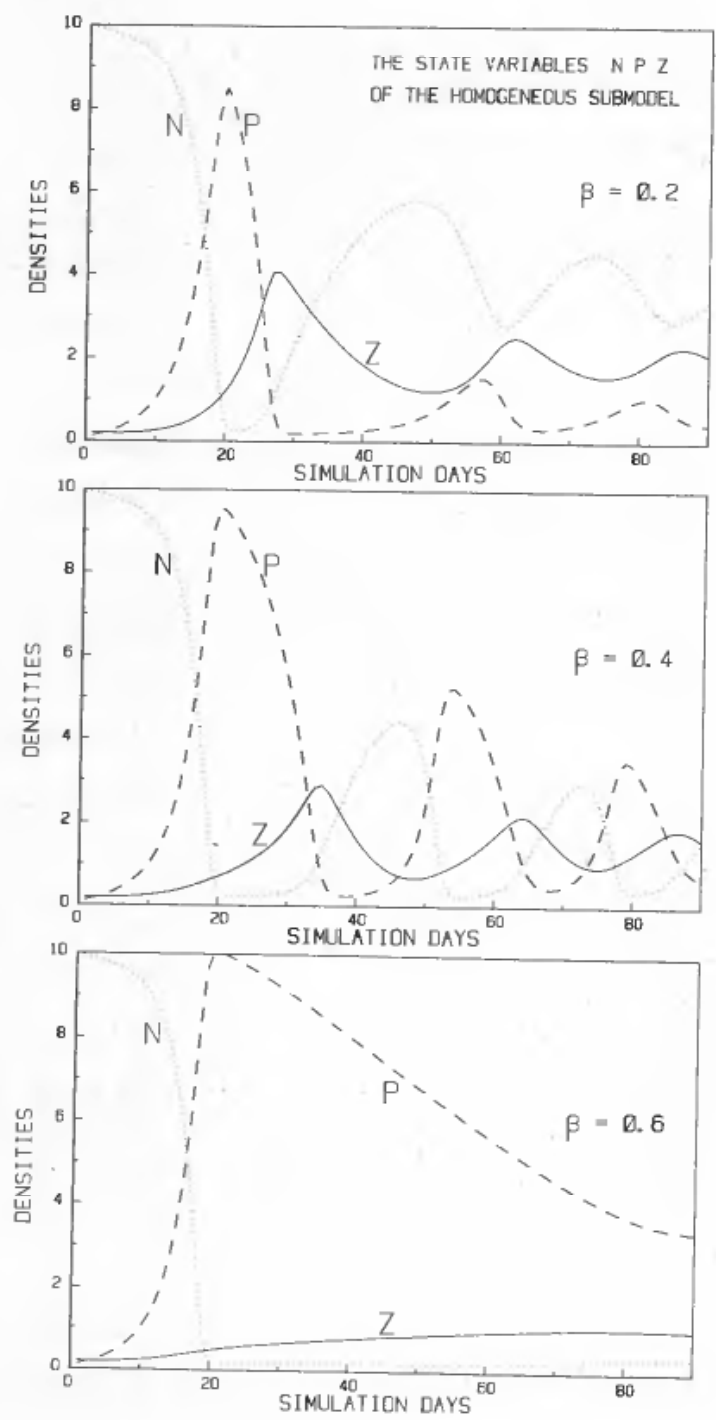

Figure 3. The state variables $N, P, Z$ as functions of time in dependence of the zooplankton mortality $\beta$. This dependence is strongly nonlinear because the character of the steady state changes with $\beta$ (see Fig. 2).

\subsection{Schools of fish}

The fish that feed on plankton often assemble in schools. Hence, a large percentage of the zooplankton mortality is very inhomogeneously distributed. Further, fish are highly mobile, they visit a special area only in irregular time intervals. Seen from such a limited area, the mortality of the zooplankton due to fish appears to be irregularly time dependent. Considering this, and keeping in mind the results in Figs. 2 and 3, in the composed model the zooplankton mortality is divided into a homogeneous part $\beta_{\mathrm{c}}$ (due to carnivorous zooplankton) and into an inhomogeneous part (due to plankton-feeding fish in schools). The constant mortality $\beta$ in the isolated submodel 
(eqn. (4)) has to be substituted by a time dependent expression:

$$
\beta_{i j}(t)=\beta_{\mathrm{c}}+\beta_{\mathrm{F}} \frac{n}{n_{\mathrm{s}}} \boldsymbol{F}_{i j}(t)
$$

The integers $i, j$ denote the position of the subsystem in the array of $n=400$ subsystems. In that array, $n_{\mathrm{s}}$ schools of fish swim around and feed on plankton. The $F_{i j}(t)$ are yes-no-functions, which tell whether or not a school of fish is in the submodel $(i, j)$ on time $t$. The time average of $F_{i j}(t)$ is just $n_{\mathrm{s}} / n$, hence, in the time average, eqn. (7) becomes eqn. (4). The fraction $q_{F}=\beta_{F} / \beta$, that is due to schools of fish, will be used as a parameter in the model calculations.

The schools are modelled to move in the model ocean from submodel to submodel, following a strategy which is determined by two parameters $Z_{F}$ and $t_{F}$ :

- The fish move to the neighbour system with the highest food concentration but they move only after the actual subsystem is grazed down below the threshold value, $Z_{F}$, and not before a minimal residence time $t_{F}$.

This strategy is an uncertain assumption. Not much is known about the behaviour of schools of fish, how they search, find and use the zooplankton patches.

It is hardly imaginable that fish do not have an essential impact on the spatial plankton distribution, although problems are connected with that idea: do fish help to create and prepare the plankton patches which they need for feeding and breeding? Furthermore, an estimation of $q_{F}$ is difficult. This number certainly depends on the kind of ecosystem and on the season. In the arctic sea, with simple food webs, and in the upwelling areas with dominant food chains, it will be much larger than in the species' rich, tropical sea, with complicated food webs (Walsh 1976, Steele 1974). A conservative estimate for $q_{F}$ in the Barents Sea will be $20 \%$ to $40 \%$.

\subsection{An amplification mechanism for plankton inhomogeneities}

The diurnal vertical migration of the zooplankton (Cushing and Walsh 1976) enables it to achieve an effective horizontal migration. The horizontal velocity components of the water movement are usually depth dependent and this speed difference between surface water and depth water can be used as a vehicle for a horizontal transport which cannot be managed by the zooplankton's own swimming capacity. A vertical migrating zooplankton patch will undergo a displacement relative to a reference point in the phytoplankton population. The displacement velocity $v$ will be around one half of the speed difference between the water layers, because the zooplankton stays roughly $12 \mathrm{~h}$ within the deeper current. An effective migration velocity $v=1 \mathrm{~km} /$ day $\simeq 1 \cdot 15 \mathrm{~cm} / \mathrm{s}$ seems to be the right order of magnitude. But contrary to the reality of changing velocities, a time independent velocity field is taken in the model.

Some care has to be taken in the numerical procedure: to avoid an artificial 'numerical' diffusion due to the zooplankton translation, the zooplankton population is shifted by one entire subsystem in time intervals $L / v$. Further, in order not to get into conflict too early on with the periodicity of the model ocean $(\S 3.1)$, in larger time intervals a shift vertical to the usual shift direction is carried out.

The effective horizontal migration of the zooplankton against the phytoplankton may have quite enormous advantages for the zooplankton:

-It serves as 'tactics for finding food' (Isaacs et al. 1974);

-A separation of age classes occurs because nauplii do not migrate so deep, hence, a food concurrence between adult and young copepodes is partly avoided. 
Remarkably enough, these advantages are only there, if the zooplankton's spatial distribution is of a patchy nature. One may interpret the situation in a new way: the zooplankton patches can be considered as predator units, with the ability of carrying out horizontal search movements in order to find the phytoplankton patches which are the prey units.

The mechanism described here is interesting in itself. In a forthcoming paper, it will be proved, for a model situation, that a horizontally homogeneous distribution of phytoplankton and zooplankton can become unstable in that way. Spontaneous creation of regular structures of the plankton densities can be observed. In reality, there are tidal currents, seasons, storms and fish prohibiting the development of regular patterns. In normal situations, the said mechanism acts as an amplifier of start inhomogeneities. Figure 4 demonstrates how, in a model situation, a hole in the zooplankton population (caused by fish) leads to an increased growth of the phytoplankton on one side of the hole and, in the following, to a zooplankton patch on one edge of the original hole.

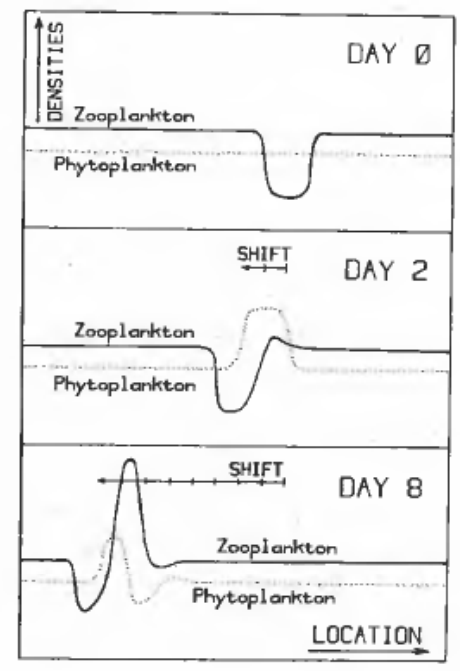

Figure 4. Amplification of an inhomogeneity in the plankton distribution in a model situation. The zooplankton moves to the left relative to the phytoplankton by diurnal vertical migration in an ocean with a corresponding velocity profile.

\subsection{Noise}

From the many mechanisms involved in the patch dynamics, one can hope to identify only a very few. In $\S \S 3.1$ to 3.3 , mechanisms have been considered that may be of prominent importance. But one cannot omit some stochastical processes. Most important in that respect is, certainly, the inhomogeneity of the vertical turbulence which supplies the nutrients. In the model, the nutrient supply can be transformed into a random process by substituting the constant $\kappa_{V}$ in eqn. (1) by a random function, dependent on time and location and, in principle, on the physical parameters. By the same token, other rate constants can be changed. In the present stage of the investigation, the stochastic element is added to the differential equations in a very simple way: at the end of each simulation day, the variables $N, P, Z$ become 
multiplied with factors $\left(1+\omega_{N} r\right),\left(1+\omega_{P} r\right),\left(1+\omega_{Z} r\right)$, where $r$ are random numbers, uniformly distributed between -1 and 1 . By changing the noise amplitudes $\omega_{N}, \omega_{P}$, $\omega_{\mathrm{z}}$, the noise part of the time-development can be made to be a minor distortion of the differential eqns. (1) to (3), or it can be made to override the deterministic part of the time course. For simplicity, and because nothing is known about the 'real' noise amplitudes, $\omega_{N}=\omega_{P}=\omega_{Z}=\omega$ is assumed.

Finally, a noise is added to the initial conditions. But an initial noise is of minor dynamic importance because it is soon smoothed away by the horizontal turbulence and, also, it is soon dominated by the dynamic noise described above.

\section{Results}

\subsection{What kind of result can be expected from the present version of the model?}

With the composed model, the dependence of the plankton patchiness on some parameters external to the homogeneous submodel can be investigated. These parameters are collated in Table 2.

As a direct output of the calculations, one obtains, for any one time-step, the full state description of all the 400 submodels. In these numbers, the detailed patchy structure of the model plankton distribution is contained. It can be represented in the form of symbolic pictures of the model ocean (Figs. 9 and 10). After successful calculations, one is in a similar situation as the experimenter, after he had done his measurements. A contraction of the information is urgently necessary, not only for the purpose of graphical representation but also for the development of patchiness variables $(\S 1.2)$. In Fig. 5, the paths of information contraction are depicted. Some forms of data representation by the computer-code for the model are given in brackets. In the left contraction branch in Fig. 5, all the information on neighbourhood relations are lost, while in the right contraction branch the density distribution details vanish. One has to go both ways parallel to their end, to enable a sufficient description of the patchiness, with few parameters, and their change with the seasons.

The movie-like representation of the time development of the patches is quite impressive but it is good for demonstration only, not for investigation.

\subsection{Diffusion and noise}

The counteraction of horizontal turbulence and noise (as defined in $\S 3.4$ ) is demonstrated most easily by the dot diagrams of Fig. 6. Here, the zooplankton density $Z$ and the phytoplankton density $P$ are represented by dots for 40 submodels and each timestep. The submodels are only coupled by the diffusion term, (eqn. 6), and disturbed by noise. No inhomogeneous zooplankton mortality is taken into account. Without diffusion, $\left(\kappa_{H}=0\right)$, a noise amplitude of $\omega=0.2$ is close to the limit where the deterministic behaviour is no longer dominant. The changes per day of $N, P, Z$ due to the differential eqns. (1) to (3) are roughly of that size ( $\simeq 20 \% /$ day). A diffusion term reduces essentially the effect of the noise term; it levels out the disturbances which are different in neighbouring subsystems. With the values $\omega=0 \cdot 2$ and $\kappa_{H}=0.05(1 \mathrm{~km}$ subsystems), diffusion and noise are reasonably balanced.

The diffusion term does, of course, not only control the noise term. It makes neighbouring subsystems look alike. That important point is discussed, together with the coherence lengths of the spatial plankton distribution, in $\S 4.6$. 


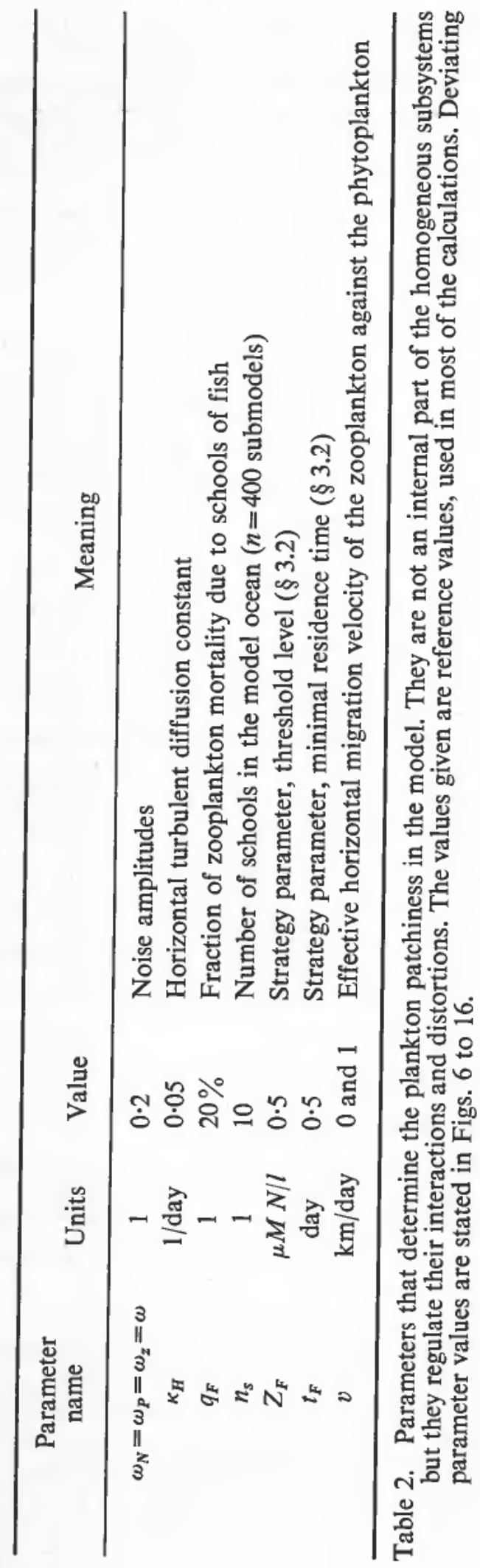




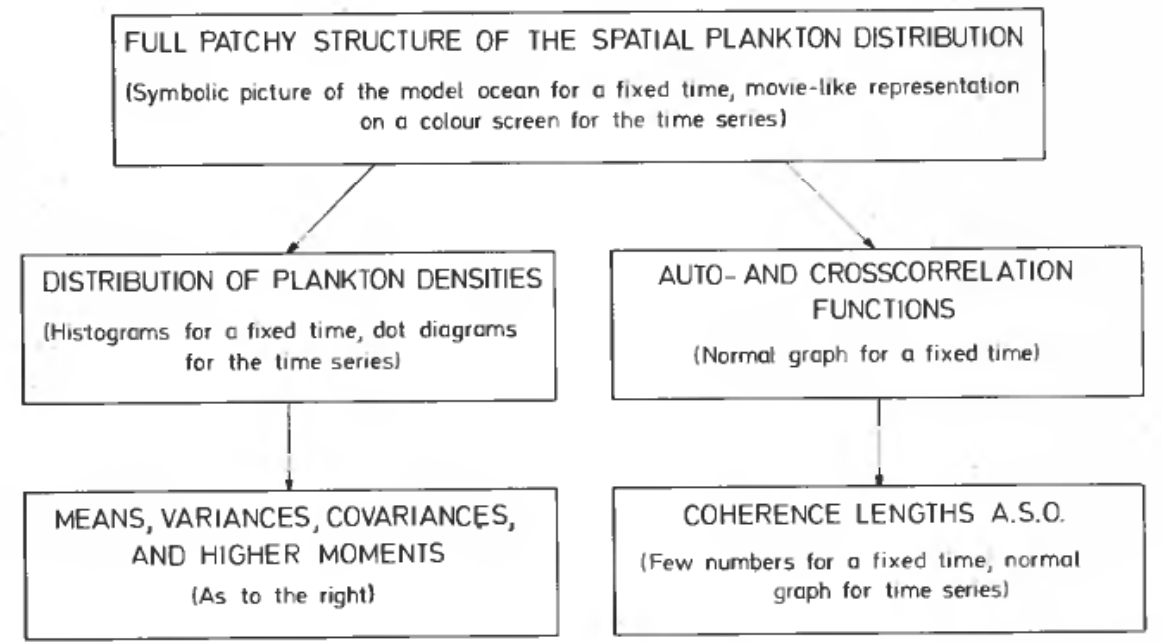

Figure 5. The paths of information contraction. In brackets: the forms of data representation by the computer code for the model.
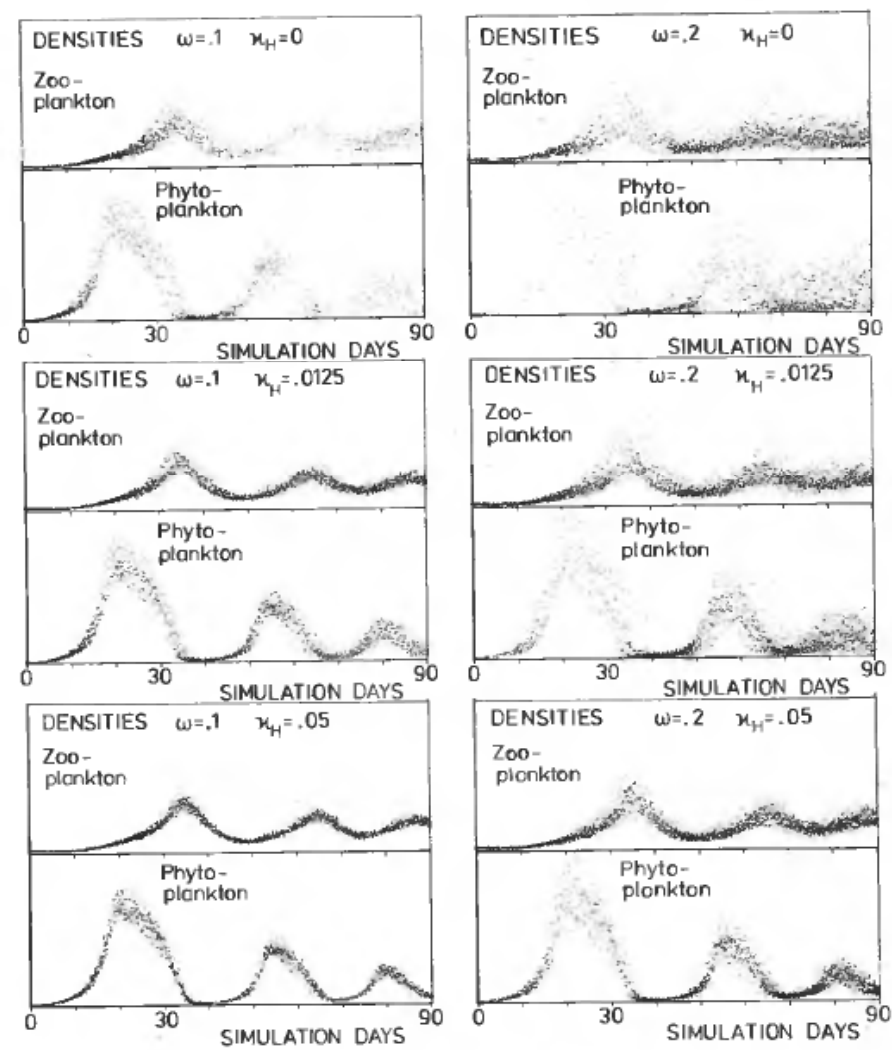

Figure 6. Plankton as functions of time (compare Fig. 3, $\beta=0.4$ ). The dispersion of the points reflects the distribution of the densities of the submodels. The scatter of points increases with the noise parameter $\omega$ and decreases with the horizontal turbulent diffusion constant $\kappa_{H}$. For $\omega=0.2$ and $\kappa_{H}=0.05$, noise and diffusion appear to be balanced. 


\subsection{Fish contribute to plankton patchiness}

The inhomogeneity of the zooplankton mortality due to moving schools of plankton-feeding fish is an important additional source of patchiness in the model (Fig. 7). The total zooplankton mortality $\beta$ is the same in all parts of Fig. 7, but the inhomogeneous fraction $q_{F}$ due to moving schools of fish is changed. To emphasize, $20 \%$ of the total mortality of 40 subsystems, concentrated in the one subsystem where the school actually resides, makes a tenfold increase of the zooplankton mortality there, compared to the mortality in the other subsystems. The fish clean up a single subsystem quite fast and move then to the next subsystem, according to the model strategy described in $\S 3.2$. The influence of the strategy parameters $Z_{F}$ and $t_{F}$ on the distribution is of a more delicate nature. Further calculations have to be done.
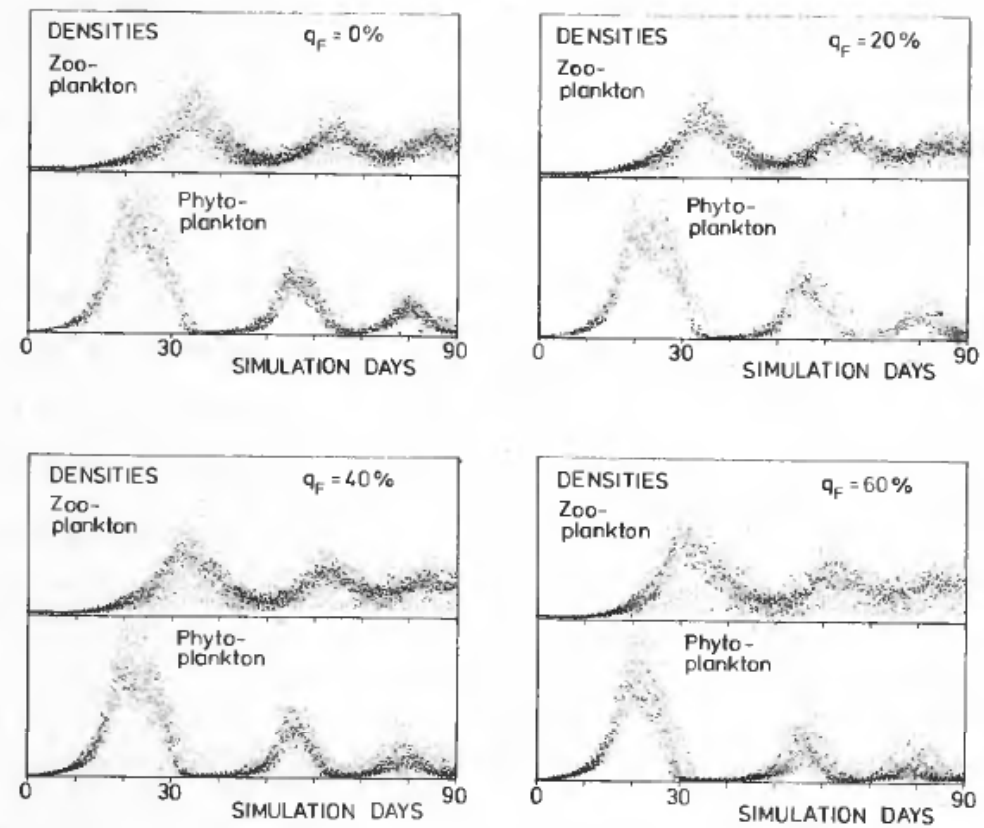

Figure 7. Plankton densities as functions of time. The dispersion of the points reflects the distribution of the densities of the submodels. It increases with the inhomogeneous fraction $q_{F}$ of the zooplankton mortality (due to schools of fish).

\subsection{The vertical migration of zooplankton and patchiness}

The central result of an effective horizontal movement of the zooplankton against the phytoplankton, $(\S 3.3)$, is the reduction of the variance of the phytoplankton and the increase of the variance of the zooplankton (Figs. 8 to 16). By its 'search movements', $(\S 3.3)$, the zooplankton diminishes the chances of a phytoplankton parch escaping the zooplankton grazing.

\subsection{The parameters of the density distributions}

Figures 9 and 10 contain a series of detailed plankton distributions of the model, corresponding to the days $28,32,48,60,80,88$ of the simulation (starting at the 

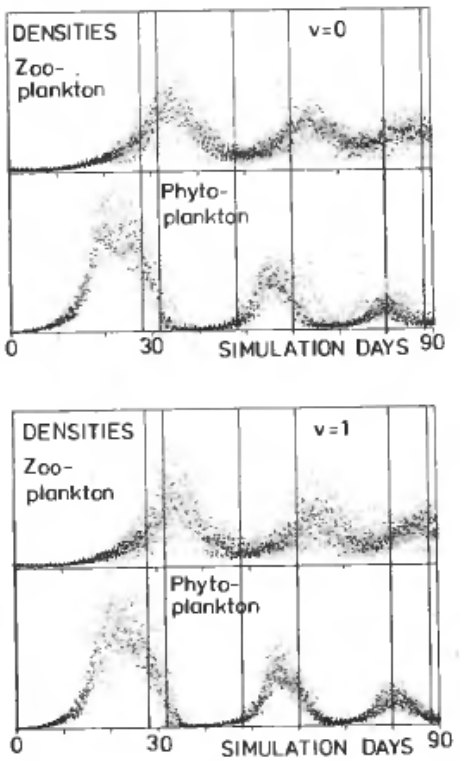

Figure 8. Plankton densities as functions of time. The dispersion of the points reflects the distribution of the densities of the submodels. With an effective horizontal motion $v$, the scatter-of the points increases for the zooplankton $Z$ and decreases for the phytoplankton $\boldsymbol{P}$. The vertical lines are reference lines for Fig. 9 and Fig. 10.

1st of March). In Fig. 8, these days are indicated by vertical lines. The character of patchiness is widely variable. Especially on later simulation days (Fig. 10), it becomes obvious that the zooplankton patchiness is more pronounced with an effective horizontal movement, $(v=1)$, then without one, $(v=0)$. This is due to the amplification mechanism, described in $\S 3.3$. One also obtains the impression of a negative correlation between phytoplankton and zooplankton patches and one has the feeling that the mean patch diameter is a few subsystems length units $L(1 \mathrm{~km})$. To transform these impressions and feelings into numbers, one has to follow the paths of information contraction, outlined in Fig. 5.

Histograms of the density distributions (Fig. 11) show strong deviations from Gaussian distributions. Hence, it is not enough to describe the density distributions by means $(\bar{P}$ and $\bar{Z})$ and variances $\left(\sigma_{P}{ }^{2}\right.$ and $\left.\sigma_{Z}{ }^{2}\right)$. At least the third moments $\left(\tau_{P}{ }^{3}\right.$ and $\left.\tau_{\mathrm{z}}{ }^{3}\right)$ are indispensable:

Densities in the single subsystem $(i, j): P(i, j)$ and $Z(i, j)$

Number of subsystems: $n=400$

$$
\begin{gathered}
\bar{P}=\frac{1}{n} \sum_{i, j} P(i, j) \\
\sigma_{P}^{2} \frac{1}{n} \sum_{i, j}(P(i, j)-\bar{P})^{2} \\
\tau_{P}^{3}=\frac{1}{n} \sum_{i, j}(P(i, j)-\bar{P})^{3}
\end{gathered}
$$

corresponding definitions for $\bar{Z}, \sigma_{Z}^{2}, \sigma_{z}{ }^{3}$. 


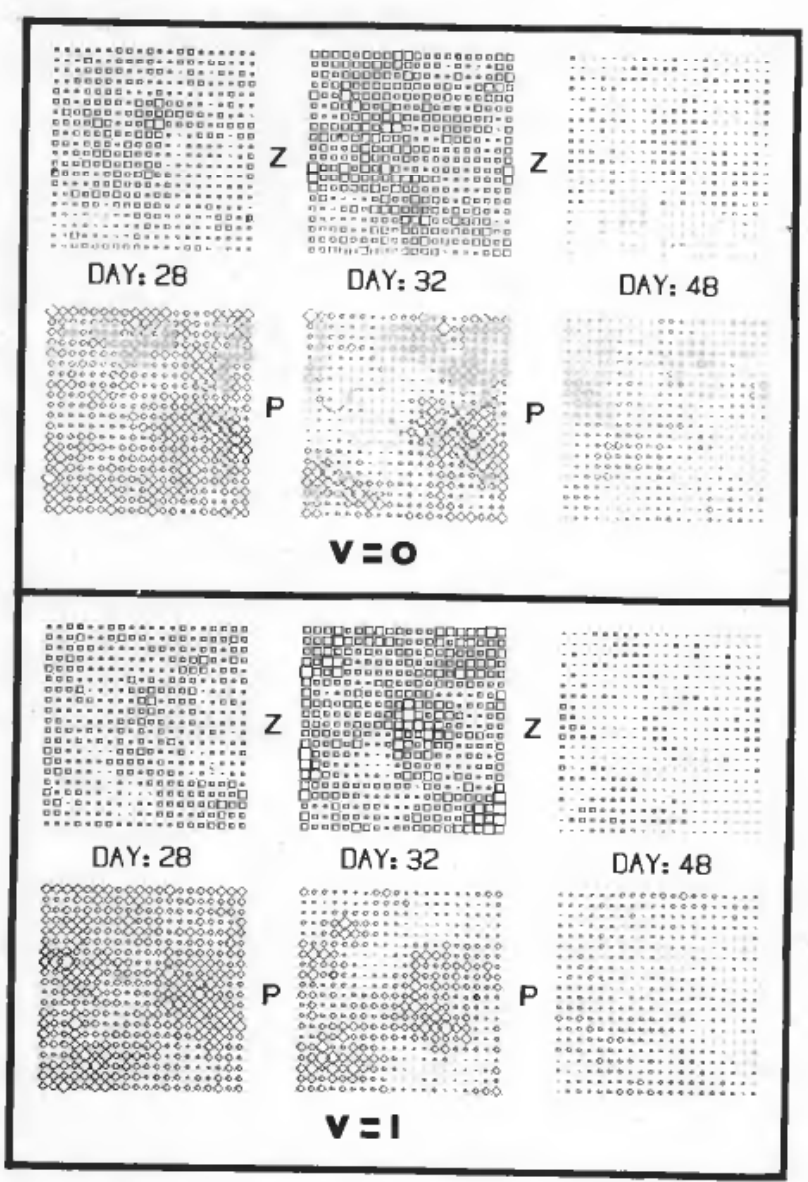

Figure 9. Symbolic representations of the model ocean. The size of the squares reflects the plankton density in the corresponding subsystem. $\square$ zooplankton, $\diamond$ phytoplankton. Simulation days: 28, 32, 48 (vertical lines in Fig. 8). At day 28, the phytoplankton densities are plotted with a reducing normalization factor $(0 \cdot 6)$. Motion: in the case $v=1$, the phytoplankton distribution is shifted one step to the left per simulation day; the zooplankton is not shifted. Ten schools of fish are there but not shown.

The time behaviour of the means of $P$ and $Z$ in Figs. 12 and 13 is quite close to the undisturbed time behaviour of an isolated subsystem (dotted). As expected, the damping of the oscillations is a bit greater. One detects clearly the increase of the moments of $Z$ and the reduction of the moments of $P$ due to $v$. There is a dramatic difference in the third moment of $Z$. While it is close to 0 for $v=0$, it becomes positive everywhere in the case of $v=1$, indicating a high density tail of the zooplankton density distribution. Such a tail occurs, if the distances between the patches exceed their diameter, that means the existence of patches in the literal sense (Fig. 10). These effects become much more pronounced (Fig. 13), if the horizontal turbulence and the noise is reduced $\left(\kappa_{H}=0.0125, \omega=0.1\right.$ instead of $\kappa_{H}=0.05, \omega=0.2$; this is again 'balanced', compare Fig. 6). As will be discussed in $\S 4.6$, this is because a lower horizontal turbulent diffusion enables more structure in an array as small as $20 * 20$ subsystems. 


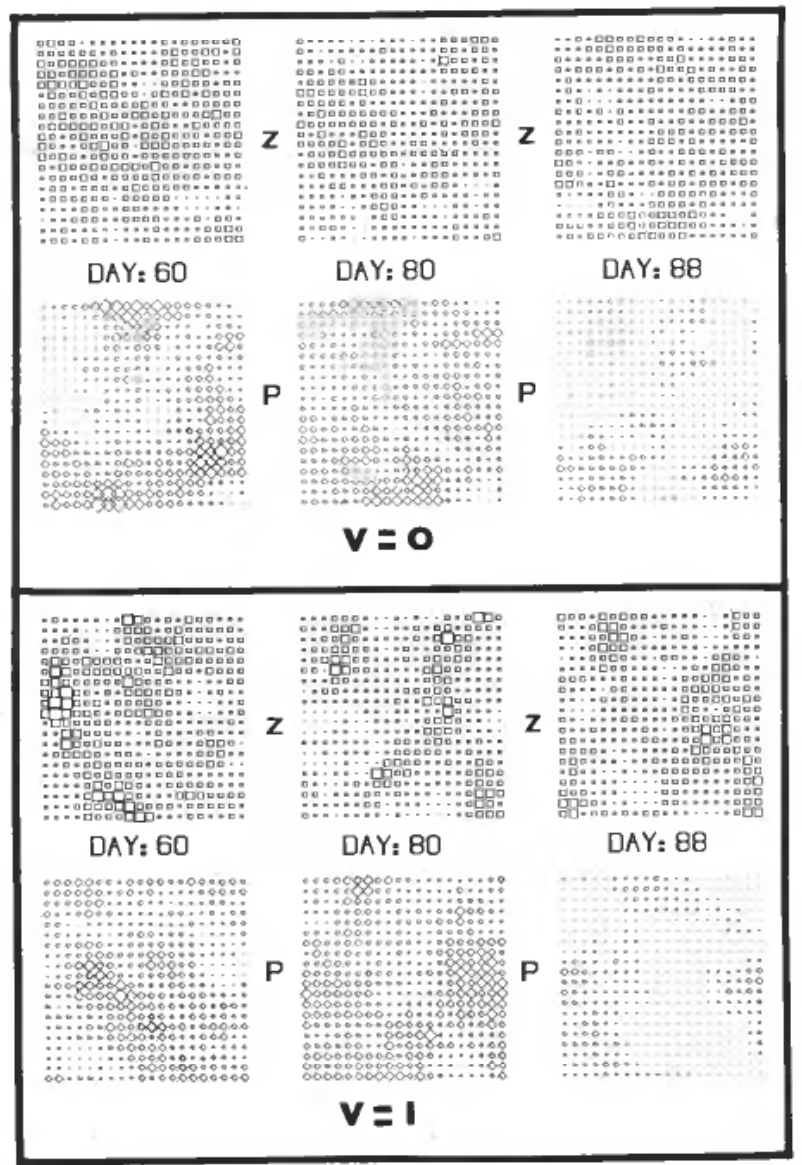

Figure 10. Same as Fig. 9, but simulation days 60, 80, 88 .

Finally in that section, the covariance $\rho$ between phytoplankton and zooplankton is considered (Fig. 14):

$$
\begin{aligned}
& \rho=\frac{1}{n} \sum_{i, j}(P(i, j)-\bar{P})(Z(i, j)-\bar{Z}) \\
& \text { correlation coefficient: } \rho / \sigma_{P} \sigma_{Z}
\end{aligned}
$$

There is generally a negative correlation between phytoplankton and zooplankton for $v=0$. But this anti-correlation becomes destroyed for $v=1$.

\subsection{The size of the patches-coherence length}

Because a patch is not a well-defined object, one cannot give a number for its diameter. A well known way to avoid this difficulty is the construction of autocorrelation functions $C_{P P}, C_{Z Z}$ and cross-correlation functions $C_{P Z}$ (Platt and Denman 1975). These functions, depending on a shift $s$ can be defined as

$$
C_{P P}(s)=\frac{1}{\sigma_{P}^{2}} \frac{1}{n} \sum_{i, j}(P(i, j)-\bar{P})(P(i+s, j)-\bar{P})
$$



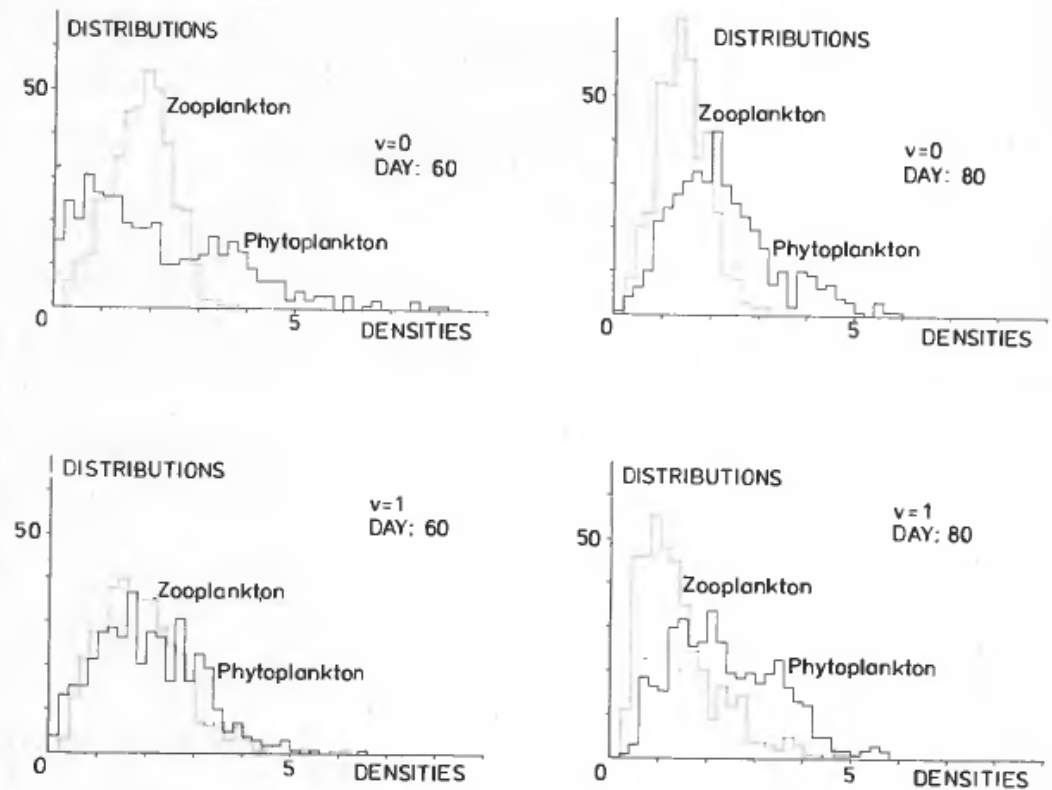

Figure 11. Plankton density distributions in the form of histograms. An effective horizontal motion $v$ causes a high density tail of the zooplankton distribution. Vertical: number of subsystems with densities in a $0 \cdot 2$-interval.
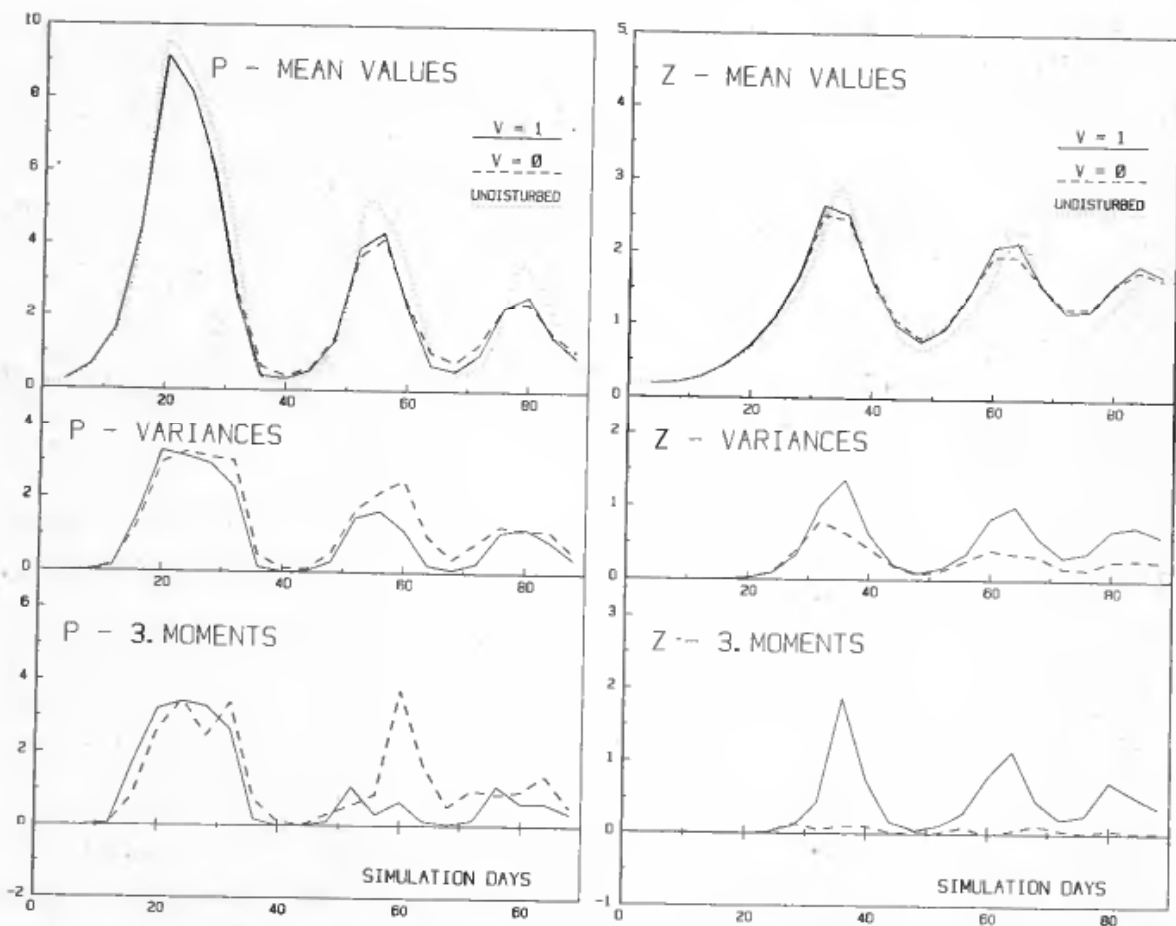

Figure 12. The moments of the plankton density distributions as functions of time. An effective horizontal motion of the zooplankton against the phytoplankton increases the zooplankton moments. The means are close to the undisturbed time behaviour (dotted, compare Fig. 3, $\beta=0 \cdot 4$ ). Parameter values from Table 2. 

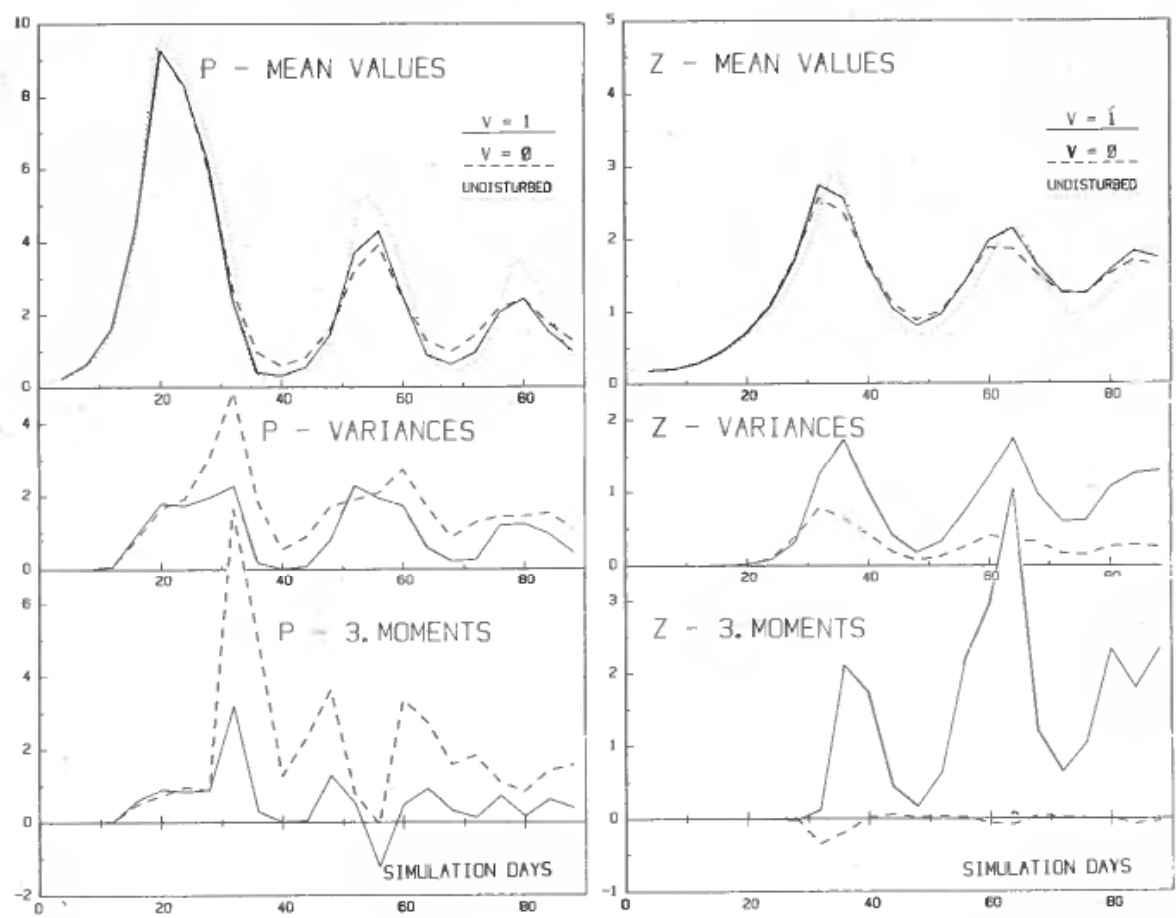

Figure 13. Same as Fig. 12, but reduced noise and turbulence $\left(\omega=0 \cdot 1, \kappa_{H}=0 \cdot 0125\right)$.

$$
\begin{aligned}
& \tilde{C}_{P P}(s)=\frac{1}{\sigma_{P}^{2}} \frac{1}{n} \sum_{i, j}(P(i, j)-\bar{P})(P(i, j+s)-\bar{P}) \\
& C_{P Z}(s)=\frac{1}{\sigma_{P} \sigma_{Z}} \frac{1}{n} \sum_{i, j}(P(i, j)-\bar{P})(Z(i+s, j)-\bar{Z})
\end{aligned}
$$

$C_{\mathrm{ZZ}}(s), \tilde{C}_{\mathrm{ZZ}}(s), \tilde{C}_{\mathrm{PZ}}(s)$ corresponding.

For $n, P(i, j), \bar{P}, \sigma_{P}{ }^{2}$ and so on, see eqns. (8) to (11). The shift can be in two directions: east-west, this is horizontal in Figs. 9 and 10, or north-south. The tilde on the functions differentiates between these cases. Because we assume a periodicity in the model ocean, the correlation functions are also periodic. The auto-correlation functions are, in addition, symmetric, hence, the maximal shift $s$ that one has to consider is half the period, which is 10 in these model calculations. Some examples of auto-correlation functions are presented in Fig. 15. For $v=0$, the east-west autocorrelation function (solid) should coincide with the north-south auto-correlation function (broken), but there are, of course, as in Figs. 12 to 14, notable statistical uncertainties. For $v=1$, a separation may occur. The motion is always close to the east-west direction (see § 3.3).

In a slightly arbitrary way, the coherence lengths are defined as the distances at which the auto-correlation functions have dropped to 1/e. In Fig. 15 they are indicated by rectangulars. The following qualitative statements can be made about the dependence of the coherence lengths from the parameters of the processes involved in the 


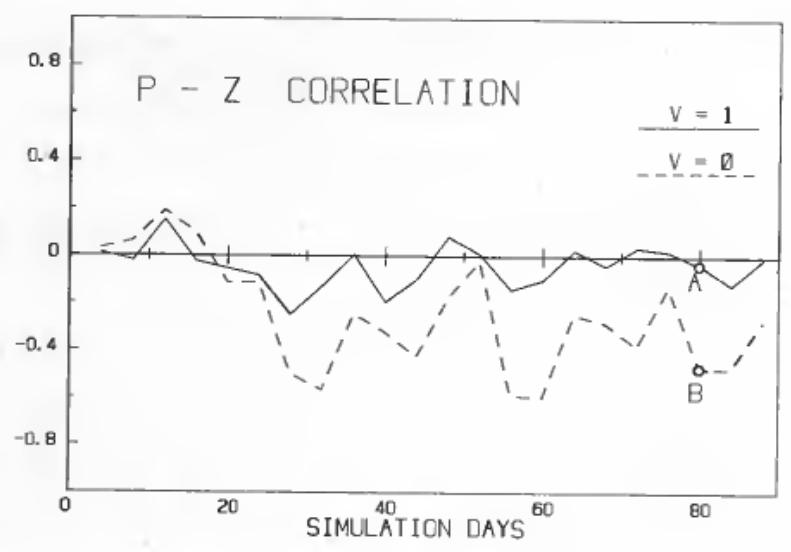

Figure 14. The correlation coefficient of phytoplankton and zooplankton as a function of time. An efficient horizontal motion destroys the anti-correlation. A and B are reference points for Fig. 16.

patchiness dynamics:

- The coherence lengths become larger for increased horizontal turbulent diffusion $\kappa_{H}$.

-A higher fraction $q_{F}$ of inhomogeneous mortality of the zooplankton (due to schools of fish) increases the phytoplankton coherence length and reduces the zooplankton coherence length.

-An effective motion $v$ increases the phytoplankton coherence length in the direction of motion and increases the zooplankton coherence length across the direction of motion.

These statements are made on the basis of many model calculations, with different parameters, but we cannot yet present numbers for these dependencies because the situation is complicated by the time dependence of the coherence lengths.

An additional problem arises from the smallness of the simulation array. On later simulation days, there is auto-correlation throughout the whole model ocean. The coherence lengths should not be too great in comparison to the subsystem diameter. Otherwise, one has to reduce $\kappa_{H}$, because the 'scale' of the diffusion process has changed (§3.1). By reducing the horizontal turbulent diffusion $\kappa_{H}$, (Fig. 13), all the results stay qualitatively unchanged but, with reduced coherence lengths, the effects due to $v$ become more pronounced.

Finally, we investigate the cross-correlation function (eqn. 14) between phytoplankton and zooplankton (Fig. 16). In the case $v=0$, the negative correlation (compare point A in Fig. 14) declines in a few steps of the shift $s$. Not so in the case $v=1$; there is no correlation for zero shift (point B in Fig. 14). Now, in the direction of motion (solid), on one side, the cross-correlation function becomes strongly negative because a zooplankton patch leaves behind a diluted lane in the phytoplankton.

\section{Conclusion}

Though the results presented in $\S 4$ are incomplete, we have shown so far:

(a) It is possible to set up a plankton model, composed from 400 homogeneous interacting submodels, to simulate the patchiness in a dynamic way. 

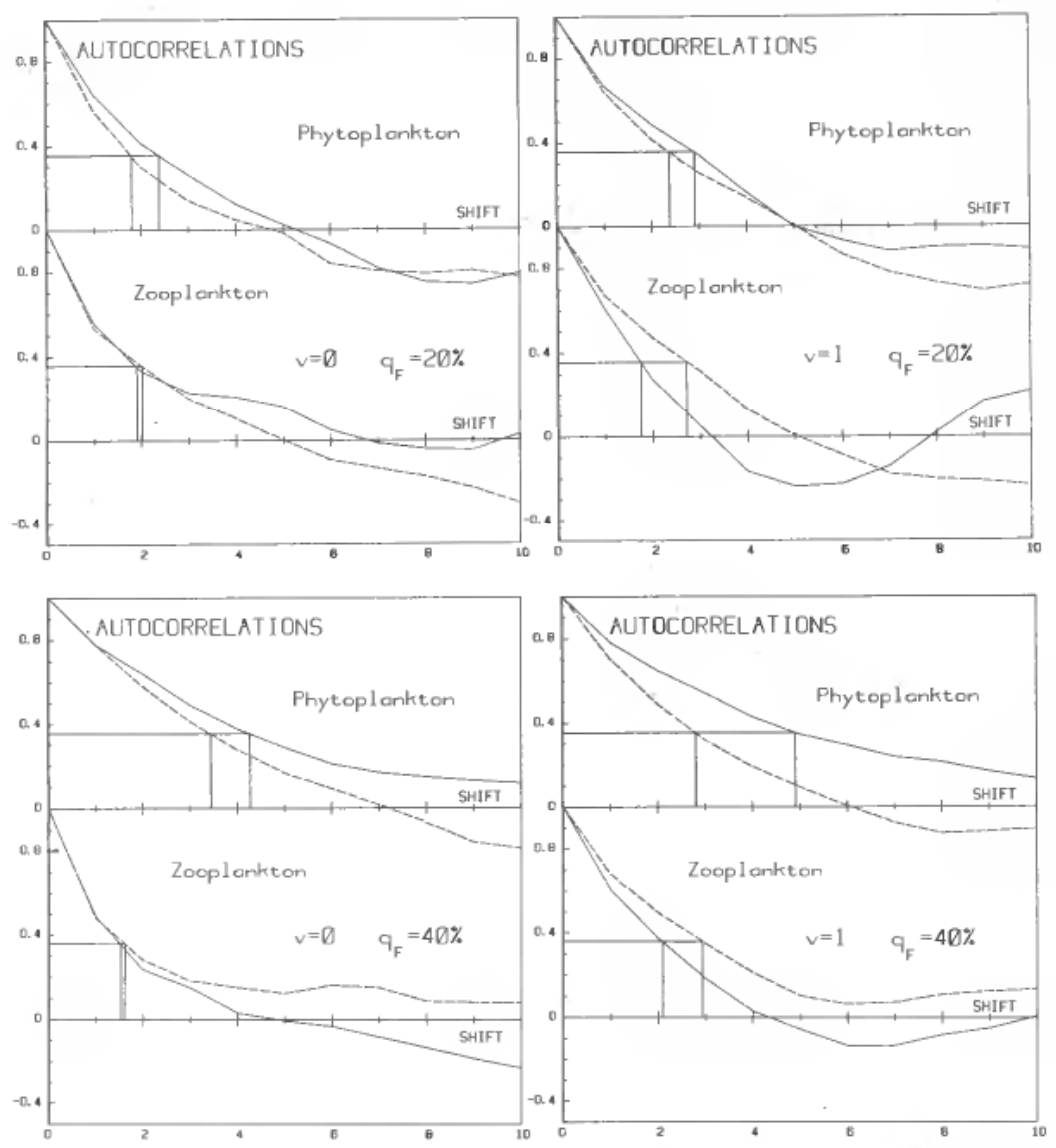

Figure 15. Auto-correlation functions on simulation day 80 (Fig. 10). In the lower diagrams, the inhomogeneous fraction $q_{F}$ of the zooplankton mortality due to schools of fish is increased. The rectangles indicate the coherence lengths. Solid lines: shift in east-west direction (direction of motion); broken lines: shift in north-south direction.

(b) Processes involved in the patch dynamics can be identified and modelled. Such processes are:

-horizontal turbulent diffusion and noise;

- the plankton consumption due to schools of fish;

-the effective horizontal motion of the zooplankton due to its vertical migration combined with currents.

(c) The counteracting pair of driving forces, noise and horizontal turbulent diffusion, can be parameterized and is, hence, open for fit procedures.

(d) Fish in schools, that feed on plankton, cause a strongly inhomogeneous mortality of the zooplankton. Not enough is known about the behaviour of fish.

(e) The vertical migration of the zooplankton leads to a horizontal shift of the migrating zooplankton population against the phytoplankton population. This mechanism reduces the phytoplankton variance and increases the zooplankton variance. 

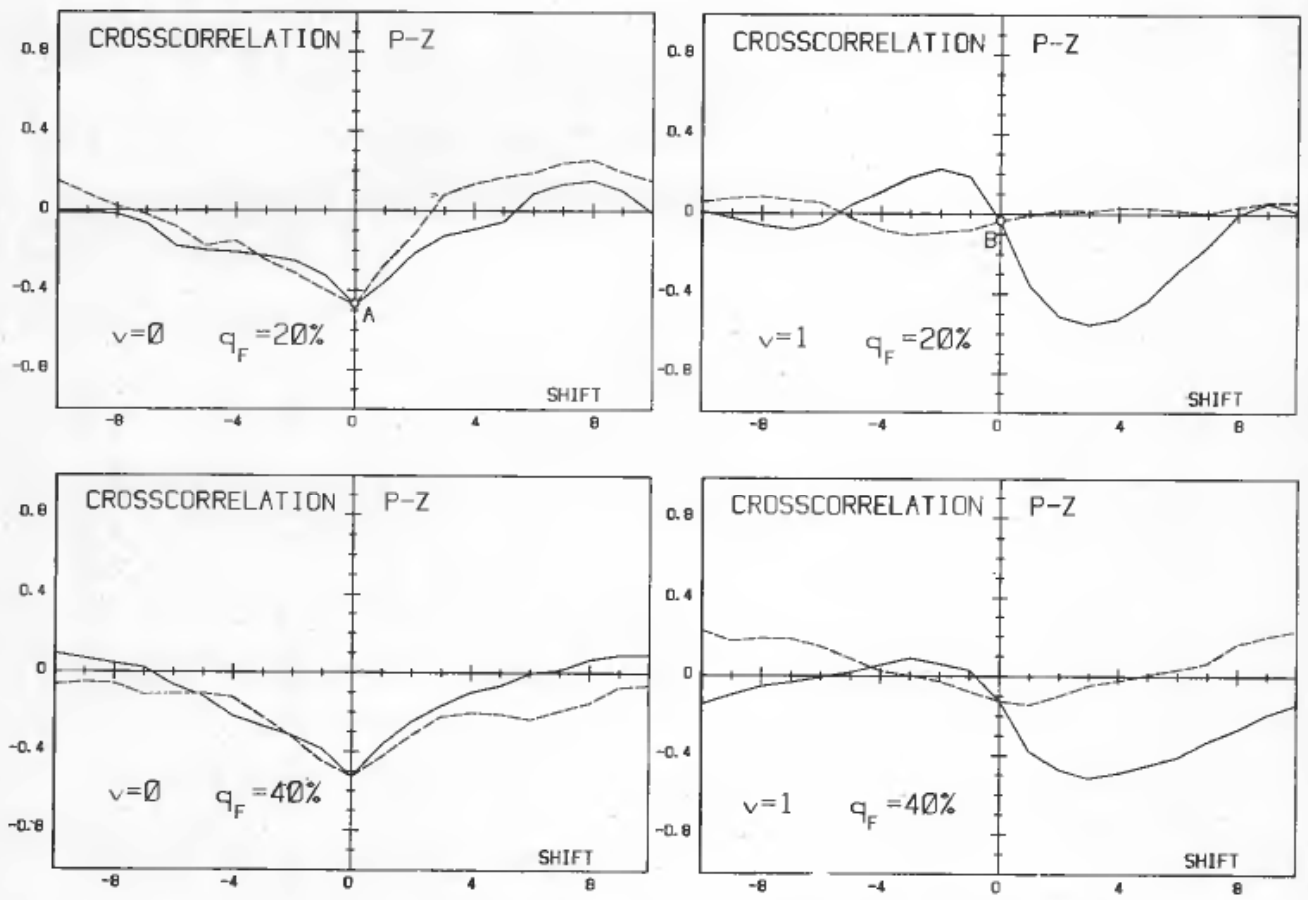

Figure 16. Cross-correlation function on simulation day 80. See note to Fig. 15. Points A and B compare with Fig. 14. An effective horizontal motion leads to an anticorrelation of phytoplankton and zooplankton on shifted locations.

$(f)$ The model leads to plankton density distributions which are non-Gaussian. The third moments, together with the coherence lengths, (see $(g)$ ), characterize the nature of the patchy structure.

(g) Coherence lengths for the spatial plankton distributions (mean distances, in that plankton densities are related) can be calculated with the model.

(h) Correlations between phytoplankton and zooplankton densities can be investigated.

The presented model is a step toward developing a dynamic patchiness model, with variables which are useful for the coupling to other subsystems of the marine ecosystem, (fish and physics), and with parameters that can be updated by measurements. But modelling of the patchiness, by simulation with a big ensemble of homogeneous subsystems, can only be a first step in the design of a patchiness model that is useful for practical applications (Balchen 1979 b).

\section{ACKNOWLEDGMENTS}

This work has been sponsored by The Royal Norwegian Council for Scientific and Industrial Research through the research program HAVBIOMODELLER (Ocean Bio-Models). The author wishes to thank Professor J. G. Balchen and D. Slagstad for the contribution of many ideas and for much helpful discussion. 


\section{REFERENCES}

BALCHEN, J. G. (1979 a). Mathematical and numerical modeling of physical and biological processes in the Barents Sea. Statistical Ecology Series, ed. G. P. Patil (Burtonsville, MD, U.S.A., International Cooperative Publishing House)

BALCHEN, J. G. (1979 b). The need for computer-aided design in modeling and control of nontechnical systems. IFAC Symposium on Computer Aided Design of Control Systems, Zürich.

Cushing, D. H., and Walsh, J. J. (1976). The Ecology of the Seas (Blackwell Scientific Publications), pp. 98 and 137.

Denman, K. L., Okubo, A., and Platt, T. (1977). The chlorophyll fluctuation spectrum in the sea. Limnol. Oceanogr., 22, 1033-1038.

Dubois, D. M. and Closset, P. L. (1975). Patchiness in primary and secondary production in the Southern Bight: a mathematical theory. 10th European Symposium on Marine Biology, Ostend, Belgium, 2, 211.

FASHAM, M. J. R. (1978). The statistical and mathematical analysis of plankton patchiness. Oceanogr. Mar. Biol. Am. Rev., 16, 43-79.

GREVE, W. (1977). Inter-specific interaction: The analysis of complex structures in carnivorous zooplankton populations. Heloländer wiss. Meeresunters., 30, 83-91.

IsAacs, J. D., ToNT, S. A., and WiCK, G. L. (1974). Deep scattering layers: Vertical migration as a tactic for finding food. Deep Sea Res., 21, 651-656.

KIERSTEAD, M., and SLOBODKIN, L. B. (1953). The size of watermasses containing plankton blooms. J. Mar. Res., 12, 141-147.

MANDELBROT, B. B. (1977). Fractals (W. H. Freeman and Company).

O'BRIEN, J. J., and WROBLEWSKI, J. S. (1973). A simulation of the mesoscale distribution of the lower marine trophic levels off West Florida. Investigation Pesquera, Barcelona, 37, 193-244.

Okubo, A. (1971). Oceanic diffusion diagrams. Deep Sea Res., 18, 789-802.

Parsons, T. R., Lebrasseur, R. J., and Fulton, J. D. (1967). Some observations on the dependence of zooplankton grazing on cell size and concentration of phytoplankton blooms. J. oceanogr. Soc. Japan, 23, 10-17.

Parsons, T. R., TAKaHAShi, M., and HARgraVe, B. (1977), Biological Oceanographic Processes, 2nd Ed. (Pergamon Press).

Platt, T. (1972). Local phytoplankton abundence and turbulence. Deep Sea Res., 19, 183-187.

Platt, T. (1975). The physical environment and spatial structure of phytoplankton populations. Mém. Soc. r. Sci. Liège, VII, 9-17.

Platt, T., and Denman, K. L. (1975). Spectral analysis in ecology. Am. Rev. Ecol. Syst., 6, 189.

Platt, T., Denman, K. L., and Jassby, A. D. (1975). The mathematical representations and prediction of phytoplankton productivity. Fisheries and Marine Services, Environment Canada, Technical Report No. 523.

Slagstad, D. (1980). A modelling of zooplankton in an ocean. Dr.ing. Thesis. Div. of Engineering Cybernetics, Norway, Inst. of Technology, Trondheim (in press).

SONNTAG, N. C., and GREve, W. (1977). Investigation of the impact of mercury on enclosed water columns using a zooplankton simulation model. J. Fish. Res. Bd of Can., 34, 2295-2307.

SteEle, J. H. (1974). The Structure of Marine Ecosystems (Harvard University Press).

WALSH, J. J. (1976). Herbivory as a factor in patterns of nutrient utilization in the sea. Limnol. Oceanogr., 21, 1.

WROBLEWSKI, J. S. (1976). A model of the spatial structure and productivity of phytoplankton populations during variable upwelling off the coast of Oregon. Florida State University, Dept. of Oceanography, Mesoscale Air-Sea Interaction Group, Techn. Report.

WroblewSKI, J. S., and O'BriEN, J. J. (1976). A spatial model of phytoplankton patchiness. Marine Biology, 35, 161-175. 